\title{
Anderson Lattice Description of Photoassociation in an Optical Lattice
}

\author{
Takahiko Miyakawa and Pierre Meystre \\ Department of Physics, University of Arizona, Tucson, Arizona 85721, USA
}

(Dated: November 21, 2018)

\begin{abstract}
We consider atomic mixtures of bosons and two-component fermions in an optical lattice potential. We show that if the bosons are in a Mott-insulator state with precisely one atom per lattice, the photoassociation of bosonic and fermionic atoms into heteronuclear fermionic molecules is described by the Anderson Lattice Model. We determine the ground state properties of an inhomogeneous version of that model in the strong atom-molecule coupling regime, including an additional harmonic trap potential. Various spatial structures arise from the interplay between the atom-molecule correlations and the confining potential. Perturbation theory with respect to the tunneling coupling between fermionic atoms shows that anti-ferromagnetic correlations develop around a spin-singlet core of fermionic atoms and molecules.
\end{abstract}

PACS numbers: 03.75.Ss, 05.30.Fk, 32.80Pj, 67.60.-g

Ultracold atoms and molecules trapped in optical lattices provide an exciting new tool for the study of strongly correlated many-body systems [1]. The exquisite degree of control of the system parameters permits the detailed study of a variety of exotic states of matter, and as a result these systems are contributing to the establishment of significant new bridges and interplay between AMO science and condensed matter physics. While much work along these lines has concentrated so far on ultracold atoms 2, 3], the coherent formation of bosonic or fermionic molecules [4, 5, 6, 7] via either Feshbach resonances [8] or two-photon Raman photoassociation [9] offers an additional path to the study of strongly correlated atoms and molecules [10, 11]. Very recently, collective coherent phenomena between an atomic and a molecular gas in an optical lattice have been observed experimentally [12].

In this letter we analyze the ground state of a mixture of atomic bosons and two-component fermions coupled to heteronuclear fermionic molecules [13, 14] by photoassociation or Feshbach resonance in an optical lattice. We show that this system can be mapped onto the Anderson Lattice Model (ALM), a model that has previously found important applications in the description of heavy electrons and intermediate valence systems in condensed matter physics. In particular, this model is known to exhibit a great variety of possible behaviors, such as e.g. the Kondo effect and magnetic ordering [15].

In the context of AMO experiments, the modifications of the ground-state properties due to the presence of a trapping potential are of particular interest. For example, in the bosonic (fermionic) Hubbard model, that potential is known to result in the coexistence of Mottinsulator and superfluid (metal) phases [16, 17]. In the strong atom-molecule coupling regime under consideration here, the ALM exhibits two types of magnetically correlated states of fermionic spins on the lattice, an on-site spin-singlet (paramagnetic) state of the atoms and molecules, and an anti-ferromagnetic (AF) corre- lated state among lattice fermionic spins. The inhomogeneity of the confining potential gives rise to a spatial structure of coexisting paramagnetic and AF domains.

We consider at zero temperature a mixture of atomic bosons of mass $M_{b}$ and atomic fermions of mass $M_{a}$ and spin $\sigma=\uparrow, \downarrow$ trapped in an optical lattice potential [18]. The fermionic and bosonic atoms can be coherently combined into heteronuclear fermionic molecules of mass $M_{m}=M_{b}+M_{a}$ via two-photon Raman photoassociation. The lattice lasers are tuned so that the fermionic atoms experience a weaker on-site lattice trapping frequency, $\Omega_{a}$, than the bosonic atoms and the fermionic molecules, $\Omega_{b}, \Omega_{m} \gg \Omega_{a}$.

The Hubbard-type Hamiltonian describing this system is $\hat{H}=\sum_{i}\left(\hat{H}_{0 i}+\hat{H}_{I i}\right)+\sum_{\langle i j\rangle} \hat{W}_{i j}$, where

$$
\begin{aligned}
\hat{H}_{0 i} & =\sum_{\sigma}\left\{\epsilon_{i \sigma}^{a} \hat{a}_{i \sigma}^{\dagger} \hat{a}_{i \sigma}+\left(\epsilon_{i \sigma}^{m}+\nu\right) \hat{m}_{i \sigma}^{\dagger} \hat{m}_{i \sigma}\right\}+\epsilon_{i}^{b} \hat{b}_{i}^{\dagger} \hat{b}_{i} \\
& +U_{a} \hat{n}_{i \uparrow}^{a} \hat{n}_{i \downarrow}^{a}+U_{m} \hat{n}_{i \uparrow}^{m} \hat{n}_{i \downarrow}^{m}+\frac{1}{2} U_{b} \hat{n}_{i}^{b}\left(\hat{n}_{i}^{b}-1\right), \\
\hat{H}_{I i} & =g \sum_{\sigma}\left(\hat{m}_{i \sigma}^{\dagger} \hat{b}_{i} \hat{a}_{i \sigma}+\text { H.c. }\right), \\
\hat{W}_{i j} & =\sum_{\sigma}\left(-t_{a} \hat{a}_{i \sigma}^{\dagger} \hat{a}_{j \sigma}-t_{m} \hat{m}_{i \sigma}^{\dagger} \hat{m}_{j \sigma}\right)-t_{b} \hat{b}_{i}^{\dagger} \hat{b}_{j}+\text { H.c. }(3)
\end{aligned}
$$

Here $\hat{a}_{i \sigma}, \hat{m}_{i \sigma}$, and $\hat{b}_{i}$ are the annihilation operators of the fermionic atoms and molecules and of the bosonic atoms at the $i$-th site, respectively. The corresponding number operators $\hat{n}_{i \sigma}^{a}=\hat{a}_{i \sigma}^{\dagger} \hat{a}_{i \sigma}, \hat{n}_{i \sigma}^{m}=\hat{m}_{i \sigma}^{\dagger} \hat{m}_{i \sigma}$, and $\hat{n}_{i}^{b}=\hat{b}_{i}^{\dagger} \hat{b}_{i}$ have eigenvalues $n_{i \sigma}^{a}, n_{i \sigma}^{m}$, and $n_{i}^{b}$.

The on-site single-particle energies $\epsilon_{i \sigma}^{\alpha}$, where $\alpha=$ $a, b, m$, consist of the sum of the contributions $\epsilon^{\alpha} \approx \Omega_{\alpha}$ from the lattice and $V_{i, \sigma}^{\alpha}$ from the trap potential at site $i$, the two-photon detuning between the Raman-lasers and the difference in internal energies between the atoms and molecules being included in $\nu$. In case the external potential is created by optical means, the spin structure of the fermions is not resolved, resulting in spin-independent single particle energies. This is the case that we consider here. The terms proportional to $U_{a}, U_{m}$, and $U_{b}$ 
describe on-site two-body interactions, inter-species collisions being ignored for simplicity. Finally, the interaction Hamiltonians $\hat{H}_{I i}$ describes the conversion of fermionic and bosonic atoms into fermionic molecules, and tunneling between nearest neighbor sites denoted by $\langle i j\rangle$ is described by the parameters $t_{a}, t_{m}$, and $t_{b}$.

We concentrate on the case $U_{b, m} \gg g \gg t_{b, m}$ and $g \gtrsim t_{a} \gg U_{a}$, a regime that is achieved for $\epsilon^{b, m} \gg \epsilon^{a}$ and $M_{b} \gg M_{a}$. One can then ignore intersite tunneling of the fermionic molecules and bosonic atoms, whose rate is given by the exponential of the ratio between the lattice constant $\lambda / 2$ and the width of the localized state $l_{\alpha}=\left(\hbar^{2} / M_{\alpha} \epsilon_{\alpha}\right)^{1 / 2}$ [11. We assume the opposite condition for the fermionic atoms and neglect instead their on-site two-body collisions 22. Typical parameters for ${ }^{87} \mathrm{Rb}$ atoms are $\epsilon_{b} / \hbar \simeq 10^{5} \mathrm{~s}^{-1}$ and $U_{b} / \hbar \simeq 10^{4} \mathrm{~s}^{-1}$, and the photoassociation coupling constant is estimated to be of the order of $g / \hbar \simeq 10^{3} \mathrm{~s}^{-1}$ []]. Since heteronuclear diatomic molecules are polar, we also need an estimate of their electric dipole-dipole interaction. The dipole moment of the triplet ${ }^{3} \Sigma^{+}$state of the $\mathrm{KRb}$ dimer is 0.02 a.u. [20], ( 1 a.u. is $\left.8.478 \times 10^{-30} \mathrm{Cm}\right)$, in which case the dipole-dipole interaction energy is less than $10 \mathrm{~s}^{-1}$ for $\lambda=1064 \mathrm{~nm}$ and can safely be ignored. We further neglect all loss processes.

In the absence of photoassociation, the ground state of the atomic fermions consists of free fermions in the confining trap $V_{i}^{a}$ while for bosons it is a Mott-insulator core with exactly one boson per site within a set $\{\ell\}$ of sites from the center of trap up to edges determined by the number of bosons $N_{b}$, and zero occupation outside. We therefore have

$$
\sum_{\sigma} \hat{n}_{i \sigma}^{m}+\hat{n}_{i}^{b}=1 \text { for } i \in\{\ell\} ; \quad \sum_{i \sigma} \hat{n}_{i \sigma}^{f}=N_{f},
$$

where $\hat{n}_{i \sigma}^{f}=\hat{n}_{i \sigma}^{a}+\hat{n}_{i \sigma}^{m}$. The former constraint is due to prohibition of double occupations and neglect of tunnelings for bosonic atoms and fermionic molecules [23]. These restrictions result in the reduced Hamiltonian

$$
\begin{aligned}
\hat{H}_{r} & =-t_{a} \sum_{\langle i j\rangle, \sigma}\left(\hat{a}_{i \sigma}^{\dagger} \hat{a}_{j \sigma}+\text { H.c. }\right)+\sum_{i \sigma} \epsilon_{i}^{a} \hat{a}_{i \sigma}^{\dagger} \hat{a}_{i \sigma} \\
& +\sum_{i \sigma}\left(\nu+\epsilon_{i}^{m}-\epsilon_{i}^{b}\right) \hat{m}_{i \sigma}^{\dagger} \hat{m}_{i \sigma}+g \sum_{i \sigma}\left(\hat{m}_{i \sigma}^{\dagger} \hat{b}_{i} \hat{a}_{i \sigma}+\text { H.c. }\right) .
\end{aligned}
$$

In the homogeneous case $V_{i}^{\alpha}=0$, this Hamiltonian is equivalent to the so-called slave boson model, which under the constraints (44) reduces to the periodic ALM with infinite on-site repulsion. We note, however, that in our model the bosonic operator $\hat{b}$ corresponds to a real physical particle, in contrast to the pseudo-boson introduced in the study of the Kondo problem [15]. In the following we investigate the ground state properties of this system in the presence of a harmonic trap and for weak tunneling of the fermionic atoms. Since the atom-molecule (AM) correlation energy is order of $g$ as shown later, this is justified in the regime $g \gg t_{a}$. We proceed by first applying a counting argument for the case $t_{a}=0$ under the constraints (4), and then account for tunneling coupling in perturbation theory.

For $t_{a}=0$, the ground state is a product of lowest energy states with total fermion number $n^{f}=n^{a}+$ $n^{m}=\sum_{\sigma} n_{i \sigma}^{a}+\sum_{\sigma} n_{i \sigma}^{m}$ on each lattice site, $\left|\Phi_{0}\right\rangle=$ $\prod_{i} \mid n^{f} ;$ lowest energy $\rangle_{i}$. For lattice sites belonging to the set $\{\ell\}$, we can have $n_{f}=0, \ldots, 3$ fermions, and the corresponding normalized lowest energy states are

$$
\begin{aligned}
& \left|n^{f}=0\right\rangle_{l}=\left|0_{a}, 0_{m}\right\rangle_{l}, \\
& \left|n^{f}=1 ; \sigma\right\rangle_{l}=\alpha_{l}\left|\sigma_{a}, 0_{m}\right\rangle_{l}+\beta_{l}\left|0_{a}, \sigma_{m}\right\rangle_{l}, \\
& \left|n^{f}=2\right\rangle_{l}=u_{l}\left|2_{a}, 0_{m}\right\rangle_{l}+v_{l}\left(\left|\uparrow_{a}, \downarrow_{m}\right\rangle_{l}-\left|\downarrow_{a}, \uparrow_{m}\right\rangle_{l}\right) \\
& \left|n^{f}=3 ; \sigma\right\rangle_{l}=\left|2_{a}, \sigma_{m}\right\rangle_{l}
\end{aligned}
$$

with corresponding energies

$$
\begin{aligned}
& E_{l}(0)=0, \\
& E_{l}(1)=\epsilon_{l}^{a}+\delta_{l}-\sqrt{\delta_{l}^{2}+g^{2}}, \\
& E_{l}(2)=2 \epsilon_{l}^{a}+\delta_{l}-\sqrt{\delta_{l}^{2}+2 g^{2}}, \\
& E_{l}(3)=3 \epsilon_{l}^{a}+2 \delta_{l},
\end{aligned}
$$

where we have used the compact notations $\left|n \sigma_{a}, n^{\prime} \sigma_{m}^{\prime}\right\rangle_{l}=\left|n \sigma_{a}\right\rangle_{l} \otimes\left|n^{\prime} \sigma_{m}^{\prime}\right\rangle_{l} \otimes\left|n^{b}=1-n^{\prime}\right\rangle_{l}$ and $2 \delta_{l}=$ $\nu+\epsilon_{l}^{m}-\epsilon_{l}^{b}-\epsilon_{l}^{a}$. Here $\alpha_{l}=-g\left\{\left[\left(\delta_{l}^{2}+g^{2}\right)^{1 / 2}-\delta_{l}\right]^{2}+g^{2}\right\}^{-1 / 2}$ and $u_{l}=-\sqrt{2} g\left\{\left[\left(\delta_{l}^{2}+2 g^{2}\right)^{1 / 2}-\delta_{l}\right]^{2}+2 g^{2}\right\}^{-1 / 2}$. The fermionic atom and molecule populations at a single site are $\left\langle n_{l}^{a}\right\rangle=\alpha_{l}^{2}$ and $\left\langle n_{l}^{m}\right\rangle=\beta_{l}^{2}$ respectively for the $n^{f}=1$ manifold, and $\left\langle n_{l}^{a}\right\rangle=1+u_{l}^{2} \leq 2$ and $\left\langle n_{l}^{m}\right\rangle=2 v_{l}^{2} \leq 1$ for $n^{f}=2$. We observe that the average number of fermionic atoms gradually decreases with decreasing $\delta_{l}$, while the number of molecules increases. From Eqs. (7) and (8), we also note that the atom-molecule conversion leads to correlated states in the $n^{f}=1$ and $n^{f}=2$ manifolds, with energies lowered by an amount of the order of $g$. The state $\left|n^{f}=2\right\rangle$ corresponds to a spin-singlet state, while spin-triplet states such as ferromagnetic configuration have no coherent scattering and then are higher energy states.

To count the number of fermions and find the ground state configuration, we introduce at each lattice site $l$ the local chemical potential $\eta_{l}(M) \equiv E_{l}(M)-E_{l}(M-1)$ $(M=1,2,3)$. It corresponds to the energy needed to add one fermion to $M-1$ fermions already at that site. At the sites $i \notin\{\ell\}$, the local chemical potential is equivalent to the atomic single particle energy, $\eta_{i}^{1}=\eta_{i}^{2}=\epsilon_{i}^{a}$. We then proceed by first determining the Fermi energy $\epsilon_{F}$ for a fixed number of fermions, the fermionic occupation number $n^{f}$ at a given site being then determined by the condition $\eta_{l}\left(n^{f}\right) \leq \epsilon_{F} \leq \eta_{l}\left(n^{f}+1\right)$, where we set $\eta_{l}(0) \rightarrow$ $-\infty$ and $\eta_{l}(4) \rightarrow+\infty$ for convenience.

In order to gain insight into the ground-state properties of the system, we consider the specific case of a onedimensional, harmonic trap potential $V_{i}^{\alpha}$ for each species 

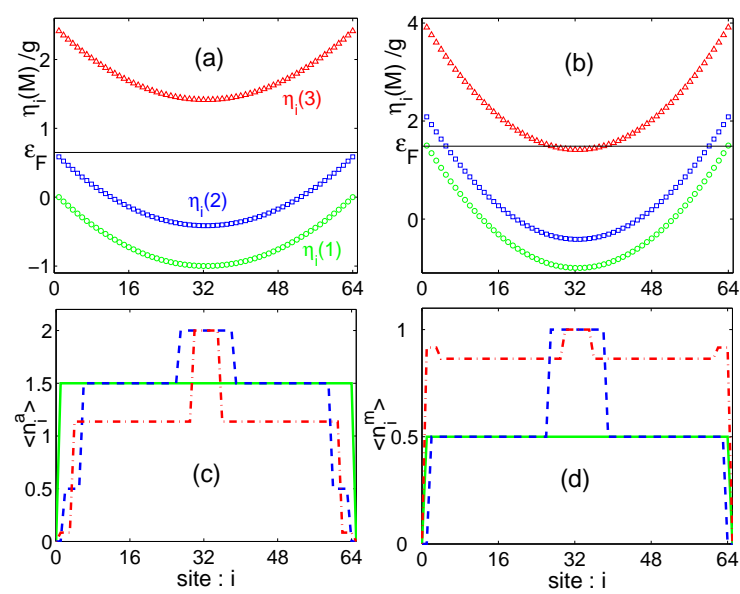

FIG. 1: (Color online) $\eta_{i}(M)$ for $\delta / g=0$ and $N_{b}=64$, and for (a) $V^{a} / g=1$, (b) $V^{a} / g=2.5$; Ground state density distribution of (c) fermionic atoms, (d) molecules for $\delta / g=0$, $V^{a} / g=1$ (solid line), $\delta / g=0, V^{a} / g=2.5$ (dashed line), and $\delta / g=-1.5, V^{a} / g=1$ (dot-dashed line) for $N_{f}=2 N_{b}=128$.

$\alpha=a, b, m$ [17], $V_{i}^{\alpha}=V^{\alpha}\left\{2 /\left(N_{b}-1\right)\right\}^{2}\left\{i-\left(N_{b}+1\right) / 2\right\}^{2}$, with the trap center located half-way between two lattice sites. We assume that the strength of molecule trapping is the sum of those of the atomic fermions and bosons, i.e., $V^{m}=V^{a}+V^{b}$, resulting in the uniform detuning $2 \delta_{l}=2 \delta \equiv \nu+\epsilon^{m}-\epsilon^{a}-\epsilon^{b}$. We also set the offset energy $\epsilon^{a}=0$ without loss of generality.

Figures 1a,b show the local chemical potentials $\eta_{l}(M)$ at each lattice site for $\delta=0$ and for (a) $V^{a} / g=1$ and (b) $V^{a} / g=2.5$. In this example, the system would contain 64 bosonic atoms occupying lattice sites within $1 \leq l \leq 64$ in the absence of photoassociation, $g=0$. We remark that in that limit all $\eta_{l}(M)$ become degenerate and equal to $\epsilon_{l}^{a}$ at $\delta=0$, clearly indicating that the gaps among the three chemical potential "bands" arise from the AM coupling. The corresponding Fermi energies for $N_{f}=2 N_{b}=128$ are the solid lines. In case (a), the ground state corresponds to a configuration where all sites within $\{\ell\}$ are in the spin-singlet state $\left|n^{f}=2\right\rangle$, all other sites being in the $\left|n^{f}=0\right\rangle$ state. In contrast, in case (b) the trap energy near the edges of the atomic density overcomes the AM correlation energy at the center of trap. As a result, the fermions are in the uncorrelated state $\left|n^{f}=3\right\rangle$ near the center of the trap and in the correlated state $\left|n^{f}=1\right\rangle$ at the edges.

The ground-state density distributions of fermionic atoms $\left\langle\hat{n}_{i}^{a}\right\rangle$ and molecules $\left\langle\hat{n}_{i}^{m}\right\rangle$ are shown in Fig. 11: and [1], respectively. For $V^{a} / g=1$ (solid line), spatially homogeneous densities of fermions are realized up to the edge of the sites $\{l\}$ and form the spin-singlet state of Eq. (8). This corresponds to the Kondo insulator [15] state in homogeneous systems. Because of the correlated gap energy this state is stable against small amounts of atomic tunneling $t_{a}$. For $V^{a} / g=2.5$ (dashed line), atoms and molecules form a spatial shell structure reminiscent of the Mott-insulator shells of bosons or fermions in combined optical lattices and trapping potentials [16, 17]. As $\delta / g$ becomes large and negative, the molecule population at each lattice site approaches unity, but the inhomogeneous character of the population persists even for $V^{a} / g=1$ at $\delta / g=-1.5$ as shown by the dot-dashed lines in Fig. 1, d.

We now discuss the effects of weak intersite tunneling of the fermionic atoms. The role of the tunneling is two folds. First, the enhancement of fluctuations of fermion number on the border between different $n^{f}$ shells. Second, the anti-ferromagnetic $(\mathrm{AF})$ correlation on the $\left|n^{f}=1 ; \sigma\right\rangle$ domain. In the strong AM coupling regime, $g \gg t_{a}$ and $|\delta| \lesssim g$, these can be investigated by (quasi)degenerate perturbation theory up to second order in $t_{a}$. The ground state is given by

$$
\left|\Psi_{g}\right\rangle=\sum_{\left\{n_{i}^{f}, \sigma_{i}\right\}} C_{g}\left\{n_{i}^{f}, \sigma_{i}\right\}\left|\Phi_{0}\left\{n_{i}^{f}, \sigma_{i}\right\}\right\rangle
$$

The set of $\left\{n_{i}^{f}, \sigma_{i}\right\}$ contains those fermion number configurations, $\left\{n_{i}^{f}\right\}$, which can be realized through multiple tunneling with an only energy cost $\left|V_{j}^{a}-V_{i}^{a}\right|(\ll g)$ for each process from the unperturbed ground state in the absence of $t_{a}$. A possible spin configuration $\left\{\sigma_{i}\right\}$ can couple to a different one via virtual excitations of energy lager by an amount of order $g$.

To illustrate the role of the number fluctuations we consider the case $N_{b}=16, V / g=1.5$, and $\delta=0$ with $N_{f}=22$ such that the unperturbed ground state in the system of $t_{a}=0$ with $\left|n^{f}=1\right\rangle$ domains surrounding the spin-singlet core of $\left|n^{f}=2\right\rangle$ and no $\left|n^{f}=3\right\rangle$ component. Figure 2 shows the density and number fluctuations of fermionic atoms, defined by $\left(\sigma_{i}^{a}\right)^{2}=\left(\left\langle\left(\hat{n}_{i}^{a}\right)^{2}\right\rangle-\right.$ $\left.\left\langle\hat{n}_{i}^{a}\right\rangle^{2}\right) /\left\langle\hat{n}_{i}^{a}\right\rangle$, as a function of $t_{a} / g$ for the direct diagonalization in the first-order perturbation theory. With increasing $t_{a}$ the hybridization of the different $n^{f}$ states is higher on the border between $n^{f}=1$ and $n^{f}=2$ shells, while the spin-singlet core and $\left|n^{f}=1\right\rangle$ domains remain. Consistently with this result, the number fluctuations of atoms develop strongly on around the border region and weakly on the core and surrounding regions. The reason of the suppression of fluctuations on the edge of the sites $\{l\}$ is that atomic tunneling from the edge sites into outside costs an energy $\sim g$, so that such a transition is neglected. We note that the result of Fig. 2 does not depend on qualitatively the excat value of the detuning parmeter and number fluctuations of molecules are vanishingly small for any $|\delta| \lesssim g$ as expected.

The second-order corrections of atomic tunneling between nearest neighbor sites that are both within the $\left|n^{f}=1\right\rangle$ manifold leads to an AF correlation among fermionic spins composed of mixtures of atoms and molecules. In contrast, if at least one site is in a $n^{f} \neq 1$ 

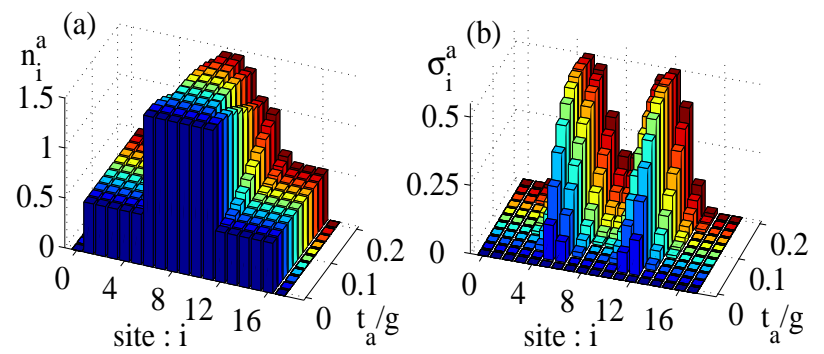

FIG. 2: (Color online) (a) $\left\langle\hat{n}_{i}^{a}\right\rangle$ and (b) $\sigma_{i}^{a}$ as a function of $t_{a} / g$ for $N_{b}=16, N_{f}=22$ and for $\delta / g=0, V^{a} / g=1.5$.

manifold, those tunneling processes are spin-independent and result only in an energy shift. The origin of AF correlation is similar to that observed in Hubbard model in the strong coupling limit [21], while in our problem at hand the coherence character between atoms and molecules plays a key role.

Introducing the coherent spin operators at the $i$-th site $s_{i, z}^{f}=(1 / 2)\left(\hat{n}_{i \uparrow}^{f}-\hat{n}_{i \downarrow}^{f}\right), s_{i,+}^{f}=\left(s_{i,-}^{f}\right)^{\dagger}=\hat{a}_{i \uparrow}^{\dagger} \hat{a}_{i \downarrow}+\hat{m}_{i \uparrow}^{\dagger} \hat{m}_{i \downarrow}$, , the spin-dependent effective Hamiltonian is

$$
\hat{H}_{s}=\sum_{\langle i j\rangle \in n^{f}=1} J_{i j}\left(\boldsymbol{s}_{i}^{f} \cdot \boldsymbol{s}_{j}^{f}-\frac{1}{4}\right)-\frac{t_{a}^{2} \alpha^{2} \beta^{2}}{\sqrt{\delta^{2}+g^{2}}} .
$$

As a consequence of AM coherence, the AF coupling coefficient $J_{i j}(>0)$ depends on the detuning parameter and, when $\left|V_{j}^{a}-V_{i}^{a}\right| \ll g, J_{i j}$ is reduced to a site-independent

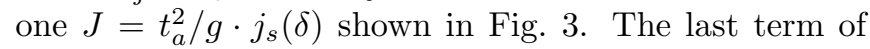
the above Hamiltonian is owing to possible tunneling between a site occupied by an atom and a neighboring one occupied by a molecule even for parallel spins.

Since Hamiltonian (11) is spin $1 / 2$ Heisenberg model, we can make out it's magnetic properties on a $\left|n^{f}=1\right\rangle$ domain. If we consider a one-dimensional example like Fig. 22 Hamiltonian (11) affects left and right sides separated by spin-singlet core independently. The ground state corresponding to each domain is characterized by $\sum_{i=1}^{L_{D}} s_{i z}^{a}=0$. The asymptotic form of the spin density correlation function has a power law behavior 21] $\left\langle s_{j+k, z}^{f} s_{j, z}^{f}\right\rangle \sim(-1)^{k} / k$ except for finite size corrections.

The present mechanism of AF correlations is unique in that atomic tunneling and strong AM couplings act cooperatively, resulting in coehrent AF characteristics among intersite fermionic spins of both atoms and molecules. Moreover, the AF correlation develops outside the paramagnetic spin-singlet core, so that the spatial paramagnetic and AF correlated domains coexist. For finding magnetic properties on the border region or when $J \sim t_{a} \ll g$, we need to consider the interplay of number fluctuations and AF correlations. This will be discussed in a future publication.

In summary, we have studied atomic boson-fermion

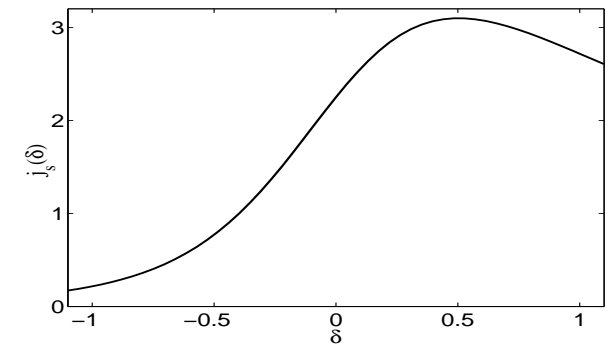

FIG. 3: Scaled AF coupling strength $j_{s}$ as a function of $\delta$.

mixtures with atom-molecule conversion in the presence of an optical lattice potential plus a harmonic oscillator potential. We have shown that by controlling lattice depths for fermions and bosons independently, an inhomogeneous version of the ALM can be realized. The new feature of inhomogeneity gives rise to various spatial structures of correlated atom-molecule ground states. We have analyzed the tunneling coupling effect by degenerate perturbation theory and have shown that this coupling and inhomogeneity give rise to coexistence of spatial domains of paramagnetic and $\mathrm{AF}$ correlations.

[1] D. Jaksch and P. Zoller, Ann. Phys. 315, 52 (2005).

[2] D. Jaksch, et al., Phys. Rev. Lett., 81, 3108 (1998).

[3] M. Greiner, et al., Nature(London) 415, 39 (2002).

[4] T. Rom, et al., Phys. Rev. Lett., 93, 073002 (2004).

[5] M. Köhl et al., Phys. Rev. Lett., 94, 080403 (2005); T. Stöferle et al., cond-mat/059211.

[6] D. Jaksch et al., Phys. Rev. Lett., 89, 040402 (2002); B. Damski et al., ibid. 90, 110401 (2003).

[7] T. Miyakawa, C. P. Search, and P. Meystre, Phys. Rev. A. 70, 053622 (2004).

[8] S. Inouye et al., Nature (London) 392, 151 (1998).

[9] R. Wynar et al., Science 287, 1016 (2000).

[10] L. D. Carr and M. J. Holland, Phys. Rev. A, 72, 031604(R) (2005).

[11] D. B. M. Dickerscheid, D. van. Oosten, E. J. Tillema, and H. T. C. Stoof, Phys. Rev. Lett. 94230404 (2005).

[12] C. Ryu et al., cond-mat/0508201

[13] C. A. Stan et al., Phys. Rev. Lett., 93, 143001 (2004).

[14] S. Inouye et al., Phys. Rev. Lett. 93, 183201 (2004).

[15] A. C. Hewson, The Kondo Problem to Heavy Fermions (Cambridge University Press, Cambridge, 1993).

[16] G. G. Batrouni et al., Phys. Rev. Lett., 89, 117203 (2002).

[17] M. Rigol, A. Muramatsu, G. G. Batrouni, and R. T. Scalettar, Phys. Rev. Lett., 91, 130403 (2003); M. Machida, S. Yamada, Y. Ohashi, and H. Matsumoto, ibid. 93, 200402 (2004).

[18] M. Lewenstein, L. Santos, M. A. Baranov, and H.Fehrmann, Phys. Rev. Lett. 92, 050401 (2004); R. Roth and K. Burnett, Phys. Rev. A 69021601 (2004); M. Cramer, J. Eisert, and F. Illuminati, Phys. Rev. Lett. 93, 190405 (2004). 
[19] K. M. O'Hara et al., Phys. Rev. A, 66, 041401(R) (2002).

[20] S. Kotochigova, P. S. Julienne, and E. Tiesinga, Phys. Rev. A 68, 022501 (2003).

[21] N. Nagaosa, Quantum Field Theory in Strongly Correlated Electronic Systems (Springer-Verlag, New York 1999).

[22] A gas of the two lowest hyperfine states of ${ }^{6} \mathrm{Li}$ is a possible candidate, where the scattering length of the mixture is very small at zero magnetic field. See, e.g., Ref. 19

[23] More rigorously, this local constraint holds in the case that the time scales of $1 / t_{b}$ and $1 / t_{m}$ are much longer than that of change of photoassociation process. In contrast, the change is assumed to be adiabatic for any other time scales including atomic tunneling $1 / t_{a}$. 